\title{
Simultaneously Enhancing Dissociation and Suppressing Recombination in Perovskite Solar Cells
}

Pei-Ying Lin ${ }^{1,2}$, Ting $\mathrm{Wu}^{1}$, Mahshid Ahmadi ${ }^{1}, \mathrm{Li} \mathrm{Liu}^{3}$, Stefan Haacke ${ }^{3}$, Tzung-Fang Guo ${ }^{2, *}$, $\mathrm{Bin} \mathrm{Hu}^{1, *}$

${ }^{1}$ Department of Materials Science and Engineering, University of Tennessee, Knoxville, Tennessee, 37996, USA

${ }^{2}$ Department of Photonics, National Cheng Kung University, 70101 Tainan, Taiwan, ROC

${ }^{3}$ Institut de Physique et Chimie des Matériaux de Strasbourg, Université de StrasbourgCNRS, 67034, Strasbourg Cedex 2, France

*Corresponding authors:

Bin Hu, E-mail: bhu@utk.edu

Tzung-Fang Guo, E-mail: guotf@mail.ncku.edu.tw 


\section{Abstract}

This article reports the experimental studies on simultaneously enhancing the dissociation and suppressing the recombination in perovskite solar cells by using high-dielectric Nickel Oxide $(\mathrm{NiOx})$ as hole transport layer. Specifically, the magneto-photocurrent, generated by the electron-hole pairs, surprisingly becomes negligible at short-circuit condition when the $\mathrm{NiOx}$ is used to replace the poly (3,4-ethylenedioxythiophene) poly (styrene-sulfonate) (PEDOT:PSS). This indicates that the NiOx transport layer leads to a complete dissociation of electron-hole pairs in perovskite layer. On the other hand, the negligible magnetophotocurrent can be recovered to become appreciable when a forward bias is applied towards open-circuit condition to weaken the built-in field. This magneto-photocurrent result suggests that the NiOx transport layer enhances the built-in field, completely dissociating the electronhole pairs. Furthermore, the photoinduced capacitance studies confirm that the built-in field is enhanced essentially through static and dynamic parameters, by removing the interfacial traps and decreasing the accumulation of photogenerated carriers. The time-resolved photoluminescence shows that the $\mathrm{NiOx} / \mathrm{CH}_{3} \mathrm{NH}_{3} \mathrm{PbI}_{3}$ interface leads to a reduction on nonradiative recombination, increasing the fraction of useful excitons available for photovoltaic actions. Moreover, the field-dependent photoluminescence measured alternatively at shortcircuit and open-circuit conditions shows that the NiOx layer can also suppress the radiative recombination within available excitons, boosting the photovoltaic actions. Therefore, our studies reveal that the high-dielectric NiOx transport layer can simultaneously enhance the dissociation of electron-hole pairs and suppress both non-radiative/radiative recombination, leading to the more efficient generation of Jsc and Voc in perovskite solar cells.

Keywords: Perovskite solar cells; Dissociation; Non-radiative/radiative recombination; Interfacial traps; Deielctric effects 


\section{Introduction}

Organo-metal halide perovskites have achieved a remarkable progress in the photovoltaic community with the certified efficiency of $22.1 \%$ in less than five years[1,2]. This rapid progress can be attributed to two major improvements. First, charge recombination has been dramatically suppressed, particularly through the non-radiative channel, by improving perovskite film quality through defect engineering [3-6]. Second, charge collection and extraction at electrode interface have been significantly improved through interface engineering by selecting ideal transport layer with high charge selectivity and appropriate energy level alignment[7-10]. Recent studies have demonstrated that the transport layer plays a major role in controlling transient photocurrent/photovoltage response and current-voltage (I-V) hysteresis phenomenon in perovskite solar cells (PSCs) [11,12]. Such I-V hysteresis phenomenon has been commonly shown in the n-i-p devices with TiOx and Spiro-OMeTAD as transport layers, which could due to serious interaction with the mobile ions, such as iodine ions/interstitials, modifying the interface barriers as well as bulk selfdegradation[11,13]. However, introducing the PCBM as either transport interlayer or bulk dopants in perovskites are shown to suppress $\mathrm{I}-\mathrm{V}$ hysteresis phenomenon due to the passivation effect of grain boundary defects[14-16]. Therefore, the inverted p-i-n perovskite solar cells with PEDOT:PSS and PCBM transport layers usually exhibit less I-V hysteresis $[12,17]$. However, the inverted perovskite solar cells usually suffer from a significant loss in the open-circuit voltage (Voc), thereby limiting the photovoltaic efficiencies. Recently, the high-dielectric Nickel Oxide $(\mathrm{NiOx})\left(\varepsilon_{r}=15\right.$ at $1 \mathrm{kHz}$ and $298 \mathrm{~K}$, refractive index $\mathrm{n}=$ 1.8 2.3 in visible light regime)[18,19] has been successfully used to replace low-dielectric PEDOT:PSS (refractive index $\mathrm{n}=1.47 \sim 1.58$ )[20] as the hole transport layer (HTL) in the inverted perovskite solar cells, which demonstrate substantial improvement on the photovoltaic performance [21-24]. This improvement has been attributed to the improved 
energy-level alignment due to the lower HOMO $(-5.4 \mathrm{eV})$ of $\mathrm{NiOx}$ as compared to the PEDOT:PSS with the higher HOMO (-5.0 -5.2 eV)[22,25]. However, it has been found that the NiOx exhibits weaker quenching effect on the photoluminescence (PL) of perovskite layer as compared to PEDOT:PSS [22,26], which seems to suggest a better charge extraction property of PEDOT:PSS that is conflict with its photovoltaic performance. Therefore, this puzzled phenomenon requires a deeper understanding on the interface associated with dielectric transport layers in perovskite solar cells towards generating a more complete guidance for future interface engineering. The early studies on organic solar cells have shown that the high-dielectric transport layer can facilitate the internal photovoltaic processes through stronger interfacial polarization [27-30]. In perovskite solar cells we can expect that a high-dielectric transport layer can have a stronger effect on internal photovoltaic processes due to the lower electron-hole binding energies in organo-metal hybrid perovskites.

In this work, we demonstrate that the NiOx HTL can simultaneously facilitate the bulk charge dissociation and suppress non-radiative/radiative recombination processes in the inverted perovskite solar cells $\left[\mathrm{ITO} / \mathrm{HTL} / \mathrm{CH}_{3} \mathrm{NH}_{3} \mathrm{PbI}_{3} / \mathrm{PC}_{61} \mathrm{BM} / \mathrm{Polyethylenimine}\right.$, ethoxylated solution (PEIE)/Ag], which can give an efficiency up to $17.53 \%(16.15 \%$ in average). By combining magneto-photocurrent measurements under external bias, timeresolved and field-dependent PL measurements, and photoinduced capacitance-voltage characterizations, we demonstrate that these synergistic effects can be attributed to the enhanced built-in field that essentially results from both the reduced interfacial traps and increased interfacial polarization at $\mathrm{CH}_{3} \mathrm{NH}_{3} \mathrm{PbI}_{3} / \mathrm{NiOx}$ interface as compared to $\mathrm{CH}_{3} \mathrm{NH}_{3} \mathrm{PbI}_{3} / \mathrm{PEDOT}$ :PSS interface. Clearly, revealing the effects of NiOx HTL on the bulk charge dissociation, recombination, and collection provides a guidance on the rational design of interface engineering towards further advancement of photovoltaic actions in organo-metal halide perovskites. 


\section{Material and methods}

\subsection{Device Fabrication}

All solar cells studied in this work were fabricated with the inverted structure of ITO/HTL/CH $\mathrm{CH}_{3} \mathrm{NH}_{3} / \mathrm{PC}_{61} \mathrm{BM} / \mathrm{PEIE} / \mathrm{Ag}$ as shown in Fig. 1(a). The energy level diagram is schematically shown in Fig. 1(b). The NiOx HTL was prepared by solution processing method according to the previous publications [22,25]. More specifically, an ethylene glycol solution containing $0.5 \mathrm{M}$ nickel formate dihydrate with 1 molar ethylenediamine was stirred overnight at room temperature and then filtered with $0.45 \mu \mathrm{m}$ nylon filters before using. The NiOx HTL was spin-cast on the precleaned ITO substrate at $4000 \mathrm{rpm}$ for 90 seconds and then annealed at $300{ }^{\circ} \mathrm{C}$ in air for 60 minutes. Alternatively, the PEDOT:PSS HTL (4083, Heraeus, filtered with $0.45 \mu \mathrm{m}$ PVDF filter) was spin-coated onto the ITO substrate at 4000 $\mathrm{rpm}$ for $60 \mathrm{~s}$ and then thermally annealed in air at $150{ }^{\circ} \mathrm{C}$ for 25 minutes. The precursor solution of $\mathrm{CH}_{3} \mathrm{NH}_{3} \mathrm{PbI}_{3}(1 \mathrm{M})$ was prepared by dissolving equimolar $\mathrm{CH}_{3} \mathrm{NH}_{3} \mathrm{I}$ and $\mathrm{PbI}_{2}$ in a mixture solvent with $\gamma$-butyrolactone (GBL) and dimethyl sulfoxide (DMSO) (7:3, v/v) at $60{ }^{\circ} \mathrm{C}$ for $12 \mathrm{~h}$ in the nitrogen-filled glove box. The perovskite films were spin-cast on top of the HTL by a consecutive two-step process at $1000 \mathrm{rpm}$ and $4000 \mathrm{rpm}$ for $20 \mathrm{~s}$ and $40 \mathrm{~s}$, respectively. The $400 \mu \mathrm{L}$ toluene was dropped on top of the perovskite films at the 20th second of the second step [31]. The as-cast films were immediately annealed at $100{ }^{\circ} \mathrm{C}$ for 10 minutes. After cooling down, the $\mathrm{PC}_{61} \mathrm{BM}$ layer $(20 \mathrm{mg} / \mathrm{mL}$ in chlorobenzene) was spin-cast on top of perovskite layer at $1000 \mathrm{rpm}$ for $60 \mathrm{~s}$ and followed by keeping the substrates in a covered Petri-dish for 45 minutes as solvent annealing. The PEIE layer (polyethyleneimine, ethoxylated solution with $0.02 \mathrm{wt} \%$ in anhydride 2-Propanol) was spin-cast on top of the $\mathrm{PC}_{61} \mathrm{BM}$ layer at $5000 \mathrm{rpm}$ for $60 \mathrm{~s}$ to modify the workfunction of $\mathrm{Ag}$. The $\mathrm{Ag}$ layer was 
thermally evaporated with the thickness of $100 \mathrm{~nm}$ under the vacuum of $6 \times 10^{-7}$ torr on the PEIE layer. The device-working area was $0.1 \mathrm{~cm}^{2}$.

\subsection{Measurements and Characterizations}

The current density-voltage $(J-V)$ characteristics of all perovskite solar cells were measured in a nitrogen-filled glovebox by using Keithley 2602 source meter under simulated sunlight (AM 1.5G, Thermal Oriel $96000300 \mathrm{~W}$, Newport). The magneto-photocurrent measurements were performed by recording the photocurrent as a function of magnetic field ( $\mathrm{B}, \sim 900 \mathrm{mT}$ ), which was applied parallel to the device plane. The B-induced photocurrent change (\%) is defined by $\frac{I(B)-I(0)}{I(0)} \times 100 \%$, where $I(B)$ and $I(0)$ represent the photocurrents with and without B, respectively. Photoinduced capacitance-voltage characteristics were measured by a dielectric spectrometer (Agilent, E4980A) with an AC bias (50 mV, $3 \mathrm{kHz}$ ). The PL spectra were recorded by SPEX Fluorolog III spectrometer under the photoexcitation of $532 \mathrm{~nm} \mathrm{CW}$ laser, while the PL lifetimes were characterized by time-resolved PL (TRPL) measurements with Streak camera (HAMAMATSU C10627). The samples for TRPL measurements were excited by the second harmonic wavelength at $515 \mathrm{~nm}$ from an Yb-doped fiber laser (TANGERINE, Amplitude System) with the fluences of $5 \mu \mathrm{J} / \mathrm{cm}^{2}$ and a repetition rate of 50 $\mathrm{kHz}$. In order to resolve the full dynamics with high resolution and signal-to-noise ratio, the dynamics of the fluorescence were recorded on several time windows, from 5 ns up to $500 \mathrm{~ns}$ with the time resolution of $0.5 \%$ for each time window. The data was fitted with exponential Eq. (1):

$$
F i t(t)=\left[\sum_{i=1}^{2} A_{i} e^{-t / \tau i}\right] \otimes \frac{1}{\sigma \sqrt{2 \pi}} e^{\left(-\frac{t^{2}}{\sigma^{2}}\right)}
$$

Where $A_{i}$ is amplitude, $\tau_{i}$ represents the lifetime, and $\sigma$ is the temporal resolution. 


\section{Results and Discussions}

\subsection{Photovoltaic Characterizations}

Fig. 1(c) presents the $J-V$ characteristics of both NiOx and PEDOT:PSS based perovskite solar cells with the key photovoltaic parameters summarized in Table $\mathbf{1}$. We can see that using the NiOx HTL to replace the PEDOT:PSS HTL leads to a large enhancement on $\mathrm{V}_{\mathrm{OC}}$ from $0.88 \mathrm{~V}$ to $1.01 \mathrm{~V}$, and simultaneously increases the short-circuit current (Jsc) from 19.32 to $21.14 \mathrm{~mA} / \mathrm{cm}^{2}$, while a high fill factor $(\mathrm{FF} \sim 0.75)$ can be readily achieved in all devices. As a result, the power conversion efficiency (PCE) is improved from $12.8 \%$ to $16.0 \%$ in average. The efficiency of the optimized devices with NiOx HTL has reached $17.53 \%$. The performance distribution over ten devices is summarized in supporting information Fig. S1. Both the NiOx and PEDOT:PSS based devices do not show any I-V hysteresis phenomenon, which suggests the high-quality of perovskite layers.

\subsection{Effects of NiOx HTL on Dissociation}

Recently, we have shown that magneto-photocurrent measurements combined with an external bias can be used as an in-situ technique to study charge dissociation and recombination in perovskite solar cells under device-operating condition $[32,33]$. This experimental method is based on the following arguments. First, the electron-hole pairs are formed in both singlets and triplets through the Coulomb capture of photogenerated carriers in organic-inorganic hybrid perovskites. Second, a magnetic field can shift the spin population between singlet and triplet electron-hole pairs[32], leading to the changes in photocurrent based on the fact that the singlet and triplet spin pairs have lower and higher dissociation rates due to allowed and forbidden transitions. Third, applying a reverse bias can facilitate the dissociation of the electron-hole pairs, quenching the magneto-photocurrent 
signal. As a result, monitoring the critical bias required to completely quench the magnetophotocurrent allows evaluating the dissociation of electron-hole pairs under device-operating condition. Fig. 2 shows the magneto-photocurrent at short-circuit condition, namely magneto-Jsc, in the NiOx and PEDOT:PSS based solar cells. Surprisingly, the NiOx-based device presents a negligible magneto-Jsc, whereas the PEDOT:PSS-based device shows an appreciable magneto-Jsc. Clearly, the negligible and appreciable magneto-Jsc imply that electron-hole pairs are sufficiently and insufficiently dissociated in NiOx and PEDOT:PSS based devices at short-circuit condition, respectively.

Interestingly, in the $\mathrm{NiOx}$-based device when applying a forward bias $(+1.0 \mathrm{~V}$, close to its Voc) to weaken the built-in field $\left(E_{\mathrm{bi}}\right)$, the magneto-Jsc is recovered to become appreciable, as shown in Fig. 3(a). On contrast, in the PEDOT:PSS-based device when applying a reverse bias $(-0.12 \mathrm{~V})$ to strength $E_{\mathrm{bi}}$, the magneto-Jsc can be completely quenched, as indicated in Fig. 3(b). This field-dependent magneto-photocurrent study provides the first evidence that the $E_{\mathrm{bi}}$ can indeed influence the charge dissociation in perovskite solar cells. More specifically, using the NiOx to replace PEDOT:PSS as HTL can enhance the charge dissociation through $E_{\mathrm{bi}}$, consequently increasing the Jsc.

\subsection{Effects of NiOx HTL on Built-in Field}

Here, we use capacitance-voltage $(C V)$ measurements in both dark and illumination conditions to evaluate the built-in potential $\left(V_{\mathrm{bi}}\right)$ in the NiOx and PEDOT:PSS based devices, respectively. Based on the $C V$ characteristics (Fig. 4 (a) and (b)), the $V_{\mathrm{bi}}$ is calculated by adopting the classic Mott-Schottky model (Eq. (2) and (3))[34,35].

$$
\begin{gathered}
C^{-2}=\frac{2\left(V_{b i}-V\right)}{A^{2} e \varepsilon \varepsilon_{0} N_{A}} \\
V_{b i}=\frac{\text { Intercept }}{\mid \text { Slope } \mid}
\end{gathered}
$$


Where the $C$ is capacitance; the $V$ is the applied bias; the $A$ is the active area; the $e$ is elementary charge of an electron; the $\varepsilon_{r}$ is the relative dielectric constant of the medium, the $\varepsilon_{0}$ is the permittivity in vacuum; and the $N_{A}$ is the density of excited states.

As shown in Fig. 4(c) and (d), the $V_{\text {bi }}$ of the NiOx and PEDOT:PSS based devices are determined to be $1.06 \mathrm{~V}$ and $0.94 \mathrm{~V}$ in dark condition and $1.02 \mathrm{~V}$ and $0.90 \mathrm{~V}$ under one-sun condition, respectively. Clearly, using the $\mathrm{NiOx}$ as HTL increases the device $V_{\mathrm{bi}}$ in both dark and illumination conditions. In general, the built-in field is determined by both the energy difference between the anode/HTL and cathode/ETL interfaces and the bulk band offset in solar cells. In organic solar cells, the bulk band offset is established with strong electrical polarization at Donor:Acceptor interface, consequently functioning as the main parameter to determine the built-in field, namely band-offset model [36]. Similarly, in perovskite solar cells the built-in field is determined by the energy difference between anode/HTL and cathode/ETL interfaces and the bulk polarization from the grain boundary defects. We should note that, when the grain boundary defects are passivated, the built-in field is mainly controlled by the energy difference between anode/HTL and cathode/ETL interfaces in perovskite solar cells, similar to metal-insulator-metal (MIM) model. This means that using different transport layer can change the built-in field in perovskite solar cells. We should further note that the anode/ETL and cathode/ETL interfaces are a function of photoexcitation due to the interfacial accumulation of photogenerated carriers. As a result, the built-in field can be divided into static and dynamic components determined by the energy difference between anode/HTL and cathode/ETL interfaces and the interfacial accumulation of photogenerated carriers. In particular, the $V_{\mathrm{bi}}$ can be largely affected by the dynamic component, namely interfacial accumulation of photogenerated carriers [27,32]. Our early studies have shown that the $V_{\text {peak }}$ shift with increasing photoexcitation intensity can reflect the interface-accumulation of photogenerated carriers in solar cells [32]. Specifically, smaller 
and larger $V_{\text {peak }}$ shifts correspond to less and more interface-accumulation of photogenerated carriers. As shown in Fig. 4(a) and (b), it is found that increasing the photoexcitation intensity causes different $V_{\text {peak }}$ shifts, $0.05 \mathrm{~V}$ and $0.12 \mathrm{~V}$ for NiOx-based and PEDOT:PSSbased devices. This suggests a reduced interface-charge accumulation when using NiOx to replace PEDOT:PSS, forming higher $V_{\mathrm{bi}}$ through a dynamic parameter in the NiOx-based device.

\subsection{Effects of NiOx HTL on Non-radiative Recombination}

In solar cells, the photogenerated carriers will inevitably undergo non-radiative and radiative recombination. The non-radiative recombination is a fast process caused by defects[37]. Through non-radiative recombination the excitons can quickly annihilate into deeply trapped carriers prior to dissociation, forming a photovoltaic loss[38-40]. In perovskite solar cells, the radiative recombination refers to a bimolecular behavior in which the excitons can be available for dissociation. Therefore, the non-radiative and radiative recombination disallows and allows the excitons for generating photovoltaic actions, respectively. Obviously, controlling the non-radiative/radiative recombination offers an important pathway to improve the photovoltaic actions in the PSCs. Fig. 5(a) shows the PL quenching of $\mathrm{CH}_{3} \mathrm{NH}_{3} \mathrm{PbI}_{3}$ layer upon applying the NiOx and PEDOT:PSS HTLs. We can see that the $\mathrm{NiOx} / \mathrm{CH}_{3} \mathrm{NH}_{3} \mathrm{PbI}_{3}$ interface leads to a weaker PL quenching but a higher Jsc as compared to the PEDOT:PSS/CH $\mathrm{CH}_{3} \mathrm{NH}_{3} \mathrm{PbI}_{3}$ interface (a stronger PL quenching but a lower Jsc). A similar phenomenon has been reported previously $[22,41]$. This puzzled phenomenon brings an important question on how the NiOx HTL can influence the non-radiative and radiative recombination in the PSCs. As illustrated in Fig. 5(b), the time-resolved PL studies suggest that the PL decay dynamics of both PEDOT:PSS/CH${ }_{3} \mathrm{NH}_{3} \mathrm{PbI}_{3}$ and $\mathrm{NiOx} / \mathrm{CH}_{3} \mathrm{NH}_{3} \mathrm{PbI}_{3}$ samples consist of fast and slow decay components, which have been ascribed to the trap- 
assisted recombination and the bimolecular recombination[42,43]. The lifetime $\left(\tau_{i}\right)$ and amplitude $\left(A_{i}\right)$, denoted in Eq. (1), determined by curve fitting are given in Fig. 5 (b) for fast and slow components. Clearly, the $\mathrm{NiOx} / \mathrm{CH}_{3} \mathrm{NH}_{3} \mathrm{PbI}_{3}$ interface yields a much longer lifetime up to $20 \mathrm{~ns}$, and fewer fast decay component (11\%); while the PEDOT:PSS/CH${ }_{3} \mathrm{NH}_{3} \mathrm{PbI}_{3}$ interface gives a much shorter lifetime of $\sim 6 \mathrm{~ns}$ and much more fast decay component (28\%). It has been demonstrated that the trap-assisted recombination is present as a non-radiative recombination channel[44,45]. Therefore, our results provide the direct information that the $\mathrm{NiOx} / \mathrm{CH}_{3} \mathrm{NH}_{3} \mathrm{PbI}_{3}$ and PEDOT:PSS/CH $\mathrm{CH}_{3} \mathrm{NbI}_{3}$ interfaces exhibit lower and higher nonradiative recombination, leading to more and less excitons available for generating Jsc, respectively. Furthermore, we should note that the suppressed non-radiative recombination upon using $\mathrm{NiOx}$ HTL suggests a reduced density of trap states at $\mathrm{HTL} / \mathrm{CH}_{3} \mathrm{NH}_{3} \mathrm{PbI}_{3}$ interface. The reduced density of trap states can be evidenced by the reduced dark current in the NiOx based device as shown in supporting information Fig. S2. Clearly, using the NiOx HTL can decrease the non-radiative recombination by reducing the interfacial traps, consequently increasing the Jsc in perovskite solar cells. We also note that a high-dielectric layer can facilitate the de-trapping of trapped carriers in organic solar cells due to interfaceinduced polarization [46], decreasing the effective density of interfacial traps. Here, the high dielectric constant may be partially responsible for the reduced interfacial trapping events in the NiOx based device.

\subsection{Effects of NiOx HTL on Radiative Recombination}

We should further note that measuring field-dependent PL can provide an evaluation on radiative recombination in perovskite solar cells [47]. In particular, the perovskite $\left(\mathrm{CH}_{3} \mathrm{NH}_{3} \mathrm{PbI}_{3}\right)$ layer possesses low electron-hole binding energies ranging from $37 \mathrm{meV}$ to 50 meV $[48,49]$. With the low binding energies, we can expect that changing the built-in field by 
applying an external bias can vary the exciton separation and consequently modifies the PL intensity. Essentially, stronger/weaker field-dependent PL reflects more/fewer excitons ready for radiative recombination. This method is based on the following arguments. First, the excitons involved in radiative recombination are available for generating photocurrent, noting that in the non-radiative recombination the excitons are not available for generating photovoltaic actions. Second, separating excitons is a field-dependent process in organometal halide perovskites due to low binding energies $[48,49]$, consequently leading to a fielddependent PL. Here, we measure the PL intensity alternatively towards short-circuit and open-circuit conditions to understand how the NiOx HTL affects the radiative recombination in the $\mathrm{CH}_{3} \mathrm{NH}_{3} \mathrm{PbI}_{3}$ layer. We can see in Fig. 5(c) that the PL intensity difference measured at short-circuit and open-circuit conditions shows larger and smaller values in the NiOx-based and PEDOT:PSS-based devices, respectively. Specifically, the PL intensity is decreased by $86 \%$ in the NiOx-based device but by $62 \%$ in the PEDOT:PSS-based device when switching from open-circuit to short-circuit condition. Equivalently, the NiOx-based device shows a stronger field-dependent PL as compared to the PEDOT:PSS-based device. Therefore, we can suggest that the NiOx HTL can also suppress the radiative recombination within available excitons under the influence of $E_{b i}$, thereby increasing the photovoltaic performance.

\section{Conclusions}

In summary, our bias-dependent magneto-photocurrent results show that using the high dielectric NiOx HTL can lead to a sufficient dissociation of electron-hole pairs in perovskite layer due to the stronger built-in field. This study reveals that the built-in field plays an important role in controlling the charge dissociation in organo-metal halide perovskites. Furthermore, our time-resolved measurement provides the direct information that the $\mathrm{NiOx}$ HTL can suppress the non-radiative recombination by decreasing interfacial traps at the 
$\mathrm{NiOx} / \mathrm{CH}_{3} \mathrm{NH}_{3} \mathrm{PbI}_{3}$ interface. Suppressing the non-radiative recombination can essentially increase the fraction of excitons available for generating photocurrent. Furthermore, we observe that the NiOx HTL leads to a larger PL change in intensity upon alternatively measured at short-circuit and open-circuit conditions, presenting a stronger field-dependent PL in the NiOx-based device. This provides an experimental indication that the NiOx HTL can also suppress the radiative recombination through the stronger built-in field, enhancing the photovoltaic actions within available excitons. Moreover, our $\mathrm{C}-\mathrm{V}$ studies confirm that the high dielectric NiOx HTL can increase the built-in field through static and dynamic parameters by reducing the interfacial traps and the interfacial accumulation of photogenerated carriers. Therefore, using the high dielectric NiOx HTL can lead to a photovoltaic enhancement through Jsc and Voc in perovskite solar cells.

\section{Acknowledgements}

This research was supported by Air Force Office of Scientific Research (AFOSR) (FA 955015-1-0064) and AOARD (FA2386-15-1-4104), and National Science Foundation (CBET1438181). This research was partially conducted at the Center for Nanophase Materials Sciences based on user projects (CNMS2012-106, CNMS2012-107, CNMS-2012-108), which is sponsored at Oak Ridge National Laboratory by the Division of Scientific User Facilities, U.S. Department of Energy. The authors (P-Y L and T-F G) acknowledge the project support from Ministry of Science and Technology (MOST) (105-2119-M-006-022MY3) in Taiwan.

\section{References}

[1] A. Polman, M. Knight, E. C. Garnett, B. Ehrler, W. C. Sinke, Science 352 (2016) 4424.

[2] NREL Efficiency Chart. http://www.nrel.gov/ncpv/images/ efficiency_chart.jpg (accessed April 19, 2016). 
[3] K. T. Cho, S. Paek, G. Grancini, C. Roldán-Carmona, P. Gao, Y. Lee, M. K. Nazeeruddin, Energy Environ. Sci. 10 (2017) 621-627.

[4] N. J. Jeon, J. H. Noh, Y. C. Kim, W. S. Yang, S. Ryu, S. I. Seok, Nat. Mater. 13 (2014) 897-903.

[5] N. D. Marco, H. Zhou, Q. Chen, P. Sun, Z. Liu, L. Meng, E. -P. Yao, Y. Liu, A. Schiffer, Y. Yang, Nano Lett. 16 (2016) 1009-1016.

[6] M. J. Simpson, B. Doughty, B. Yang, K. Xiao, Y.-Z. Ma, J. Phys. Chem. Lett. 7 (2016) $1725-1731$.

[7] H. Zhou, Q. Chen, G. Li, S. Luo, T. -B. Song, H. -S. Duan, Z. Hong, J. You, Y. Liu, Y. Yang, Science 345 (2014) 542-546.

[8] T. -Y. Chiang, G. -L. Fan, J. -Y. Jeng, K. -C. Chen, P. Chen, T. -C. Wen, T. -F. Guo, K. T. Wong, ACS Appl. Mater. Interfaces 7 (2015) 24973-24981.

[9] C. Liu, Z. Su, W. Li, F. Jin, B. Chu, J. Wang, H. Zhao, C. S. Lee, J. Tang, B. Kang, Org. Electron. : Phys. Mater. Appl. 33 (2016) 221-226.

[10] W. Chen, Y. Wu, Y. Yue, J. Liu, W. Zhang, X. Yang, H. Chen, E. Bi, I. Ashraful, M. Grätzel, L. Han, Science 350 (2015) 944-948

[11] C. Li, S. Tscheuschner, F. Paulus, P. E. Hopkinson, J. Kiessling, A. Kohler, Y. Vaynzof, S. Huettner, Adv. Mater. 28 (2016) 2446-2454.

[12] M. De Bastiani, G. Dell'Erba, M. Gandini, V. D'Innocenzo, S. Neutzner, A. R. S. Kandada, G. Grancini, M. Binda, M. Prato, J. M. Ball, M. Caironi, A. Petrozza, Adv. Energy Mater. 6 (2016) 1501453.

[13] G. Divitini, S. Cacovich, F. Matteocci, L. Cinà, A. Di Carlo, C. Ducati, Nat. Energy 1 (2016) 15012.

[14] C. -H. Chiang, C. -G. Wu, Nat. Photon. 10 (2016) 196-200.

[15] Y. Shao, Z. Xiao, C. Bi, Y. Yuan, J. Huang, Nat. Commum. 5 (2014) 5784.

[16] Z. Zhu, Q. Xue, H. He, K. Jiang, Z. Hu, Y. Bai, T. Zhang, S. Xiao, K. Gundogdu, B. R. Gautam, H. Ade, F. Huang, K. S. Wong, H. -L. Yip, S. Yang, H. Yan, Adv. Sci. 3 (2016) 1500353.

[17] H. Kim, K. -G. Lim, T. -W. Lee, Energy Environ. Sci. 9 (2016) 12-30.

[18] K. V. Rao, A. Smakula, J. Appl. Phys. 36 (1965) 2031-2038.

[19] S. A. Mahmoud, A. Shereen, M. a. A. Tarawnh, J. Mod. Phys. 02 (2011) 1178.

[20] C.-W. Chen, S.-Y. Hsiao, C.-Y. Chen, H.-W. Kang, Z.-Y. Huang, H.-W. Lin, J. Mater. Chem. A 3 (2015) 9152-9159. 
[21] W. Chen, Y. Wu, J. Liu, C. Qin, X. Yang, A. Islam, Y. -B. Cheng, L. Han, Energy Environ. Sci. 8 (2015) 629-640.

[22] J. -Y. Jeng, K. -C. Chen, T. -Y. Chiang, P. -Y. Lin, T. -D. Tsai, Y. -C. Chang, T. -F. Guo, P. Chen, T. -C. Wen, Y.-J. Hsu, Adv. Mater. 26 (2014) 4107-4113.

[23] K. -C. Wang, J. -Y. Jeng, P. -S. Shen, Y. -C. Chang, E. W.-G. Diau, C.-H. Tsai, T.-Y. Chao, H. -C. Hsu, P. -Y. Lin, P. Chen, T. -F. Guo, T. -C. Wen, Sci. Rep. 4 (2014) 4756.

[24] Y. Wu, X. Yang, W. Chen, Y. Yue, M. Cai, F. Xie, E. Bi, A. Islam, L. Han, Nat. Energy 1 (2016) 16148.

[25] A. Garcia, G. C. Welch , E. L. Ratcliff, D. S. Ginley, G. C. Bazan, D. C. Olson, Adv. Mater. 24 (2012) 5368-5373.

[26] P. Docampo, J. M. Ball, M. Darwich, G. E. Eperon, H. J. Snaith, Nat. commun. 4 (2013) 2761.

[27] T. Wu, Y. -C. Hsiao, M. Li, N. -G. Kang, J. W. Mays, B. Hu, J. Phys.Chem. C 119 (2015) 2727-2732.

[28] Q. Zhang, B. Kan, F. Liu, G. Long, X. Wan, X. Chen, Y. Zuo, W. Ni, H. Zhang, M. Li, Z. Hu, F. Huang, Y. Cao, Z. Liang, M. Zhang, T. P. Russell, Y. Chen, Nat. Photon. 9 (2015) 35-41.

[29] Z. He, B. Xiao, F. Liu, H. Wu, Y. Yang, S. Xiao, C. Wang, T. P. Russell, Y. Cao, Nat. photon. 9 (2015) 174-179.

[30] G. Li, V. Shrotriya, J. Huang, Y. Yao, T. Moriarty, K. Emery, Y. Yang, Nat. Mater. 4 (2005) 864-868.

[31] N. J. Jeon, J. H. Noh, Y. C. Kim, W. S. Yang, S. Ryu, S. I. Seok, Nat. Mater. 13 (2014) 897-903.

[32] M. Ahmadi, Y. -C. Hsiao, T. Wu, Q. Liu, Q. Wei, B. Hu, Adv. Energy Mater. 7 (2016) 1575 .

[33] Y. -C. Hsiao, T. Wu, M. Li, B. Hu, Adv. Mater. 27 (2015) 2899-2906.

[34] G. Perrier, R. de Bettignies, S. Berson, N. Lemaître, S. Guillerez, Sol. Energ. Mat. Sol. Cells 101 (2012) 210-216.

[35] F. Fabregat-Santiago, G. Garcia-Belmonte, J. Bisquert, P. Bogdanoff, A. Zaban, J Electrochem. Soc. 150 (2003) 293.

[36] N. K. Elumalai, A. Uddin, Energy Environ. Sci. 9 (2016) 391-410.

[37] A. Marchioro, J. Teuscher, D. Friedrich, M. Kunst, R. van de Krol, T. Moehl, M. Grätzel, J. -E. Moser, Nat. Photon. 8 (2014) 250-255. 
[38] K. Tvingstedt, O. Malinkiewicz, A. Baumann, C. Deibel, H. J. Snaith, Sci. Rep. 4 (2014) 6071.

[39] W. Tress, Springer International Publishing: Cham. 53-77 (2016).

[40] T. Leijtens, G. E. Eperon, A. J. Barker, G. Grancini, W. Zhang, J. M. Ball, A. R. S. Kandada, H. J. Snaith, A. Petrozza, Energy Environ Sci. 9 (2016) 3472-3481.

[41] Y. -K. Chih, J. -C. Wang, R. -T. Yang, C. -C. Liu, Y. -C. Chang, Y .-S. Fu, W. -C. Lai, P. Chen, T. -C. Wen, Y. -C. Huang, C. -S. Tsao, T. -F. Guo, Adv. Mater. 28 (2016) 86878694.

[42] Y. Yamada, T. Nakamura, M. Endo, A. Wakamiya, Y. Kanemitsu, J. Am. Chem. Soc., 136 (2014) 11610-11613.

[43] D. W. de Quilettes, S. M. Vorpahl, S. D. Stranks, H. Nagaoka, G. E. Eperon, M. E. Ziffer, H. J. Snaith D. S. Ginger, Science 348 (2015) 683-686.

[44] G. J. Wetzelaer, M Scheepers, A. M. Sempere, C. Momblona, J. Ávila, H. J. Bolink, Adv. Mater. 27 (2015) 1837-1841.

[45] J. -C. Blancon, W. Nie, A. J. Neukirch, G. Gupta, S. Tretiak, L. Cognet, L. Cognet, A. D. Mohite, J. J. Crochet, Adv. Funct. Mater. 26 (2016) 4283-4292.

[46] Hsiao Y-C, Zang H, Ivanov I, Xu T, Lu L, Yu L, et al. Dielectric Interface Effects on Surface Charge Accumulation and Collection towards High-Efficiency Organic Solar Cells. Journal of Applied Physics 115, (2014) 154506.

[47] K. Tvingstedt, O. Malinkiewicz, A. Baumann, C. Deibel, H. J. Snaith, V. Dyakonov, H. J. Bolink, Sci. Rep. 4 (2014) 6071.

[48] P. Löper, M. Stuckelberger, B. Niesen, J. Werner, M. Filipič, S. -J. Moon, J. -H. Yum, M. Topič, S. De Wolf, C. Ballif, J. Phys. Chem. Lett. 6 (2015) 66-71.

[49] V. D’Innocenzo, G. Grancini, M. J. Alcocer, A. R. Kandada, S. D. Stranks, M. M. Lee, G. Lanzani, H. J. Snaith, A. Petrozza, Nat. Commun. 5 (2014) 3586. 
Figures and Tables

(a)

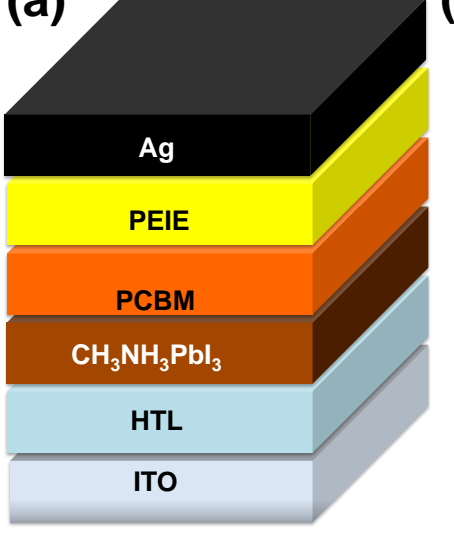

(b)

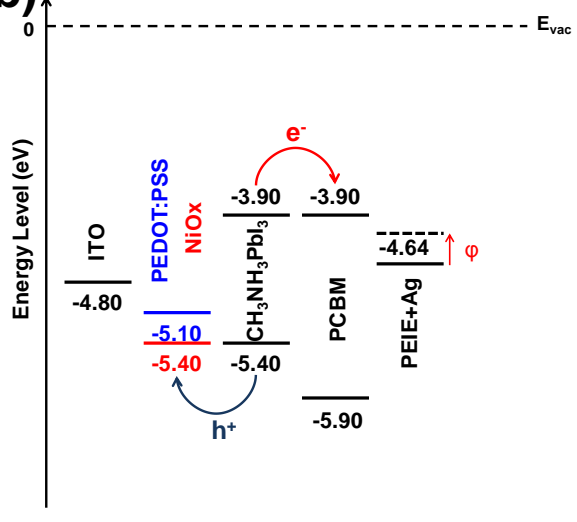

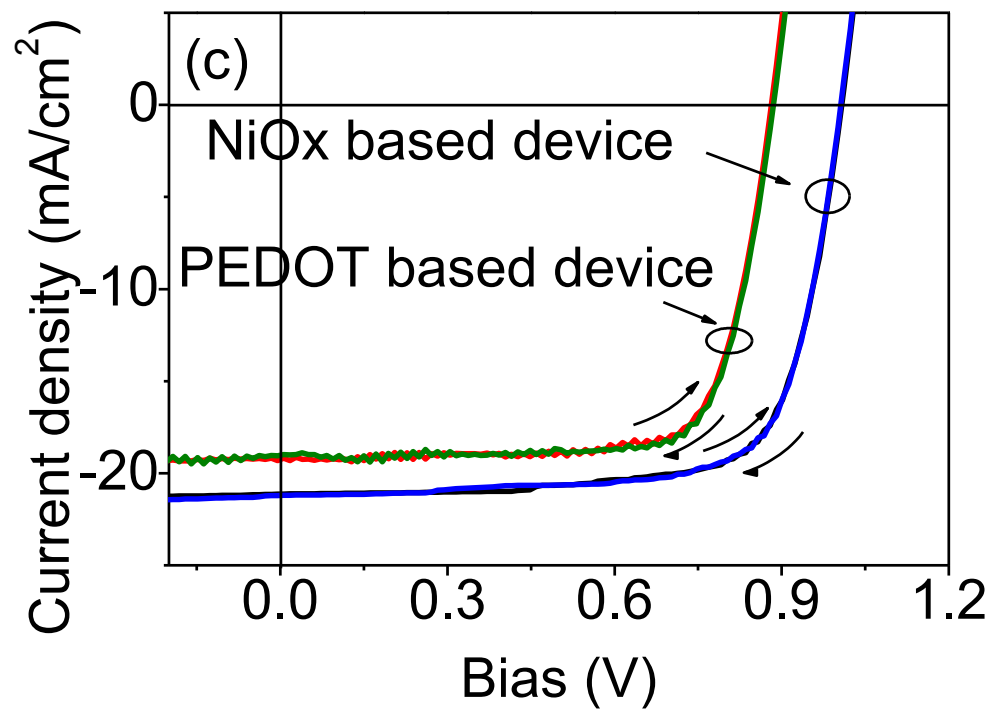

Fig. 1. a) Device architecture. The HTL refers to NiOx or PEDOT:PSS; b) Energy diagram for the fabricated devices [ITO/HTL/CH${ }_{3} \mathrm{NH}_{3} \mathrm{PbI}_{3} / \mathrm{PC}_{60} \mathrm{BM} / \mathrm{PEIE} / \mathrm{Ag}$ ]; c) $J-V$ characteristics measured under forward and reverse scans. 


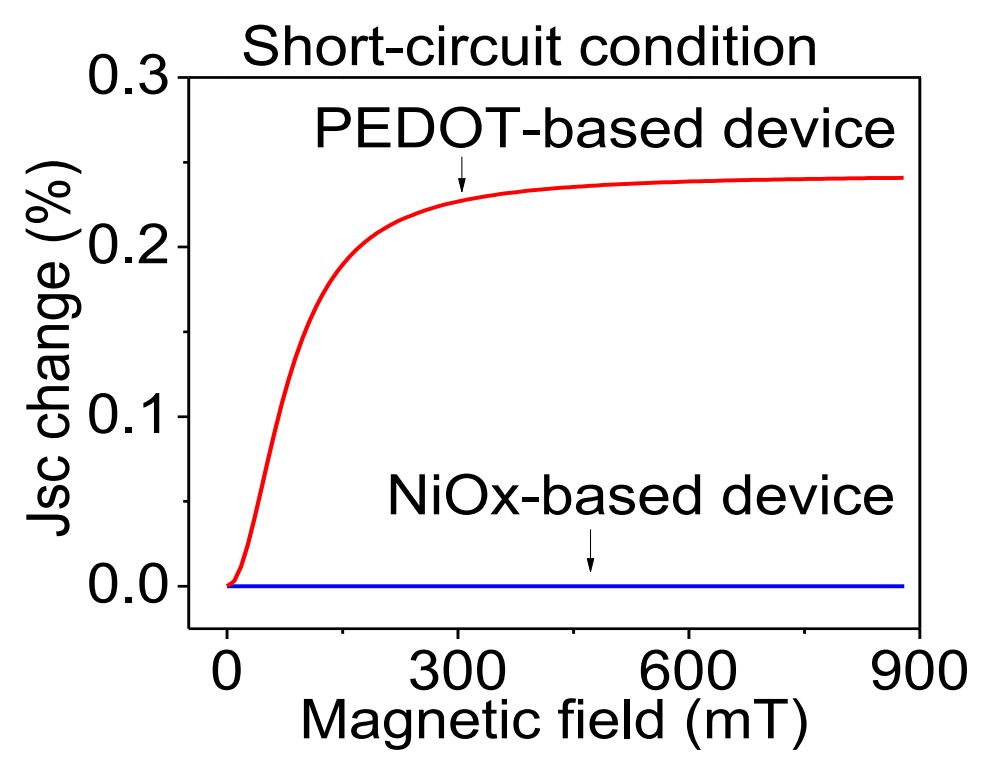

Fig. 2. Magneto-Jsc becomes negligible and appreciable in NiOx and PEDOT:PSS based devices values at short-circuit condition (zero external bias). Negligible and appreciable magneto-Jsc correspond to sufficient and insufficient dissociation of electron-hole pairs in NiOx and PEDOT:PSS based devices, respectively. 

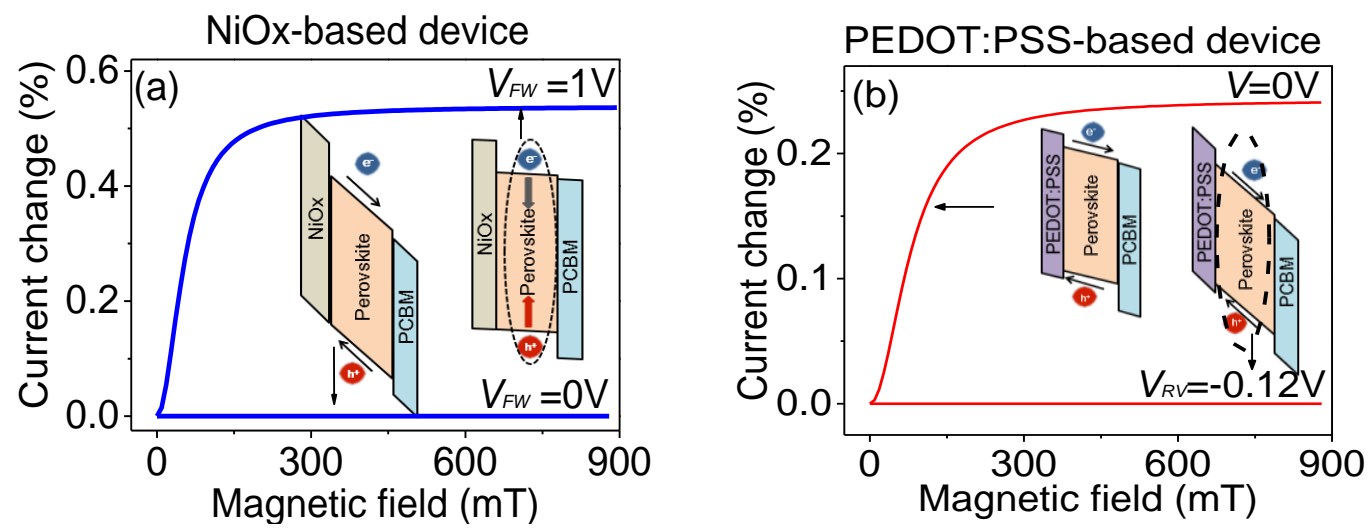

Fig. 3. Magneto-photocurrent upon adjusting built-in field by an external bias. a) In NiOxbased device magneto-photocurrent changes from negligible to appreciable value by decreasing built-in field through applying forward bias (+1.0 V); b) In PEDOT:PSS-based device magneto-photocurrent changes from appreciable to a negligible value by increasing the built-in field through applying a reverse bias $(-0.12 \mathrm{~V})$. 



Fig. 4. Capacitance-voltage $(C V)$ characteristics of solar cells with a) PEDOT:PSS and b) NiOx HTL under AM $1.5 \mathrm{G}$ simulated sunlight with various intensities. c) and d) $C^{-2}-V$ in dark condition and under one sun illumination for perovskite solar cells with PEDOT:PSS and NiOx HTLs, respectively. The built-in potential $\left(V_{\mathrm{bi}}\right)$ is derived from Mott-Schottky equation. 

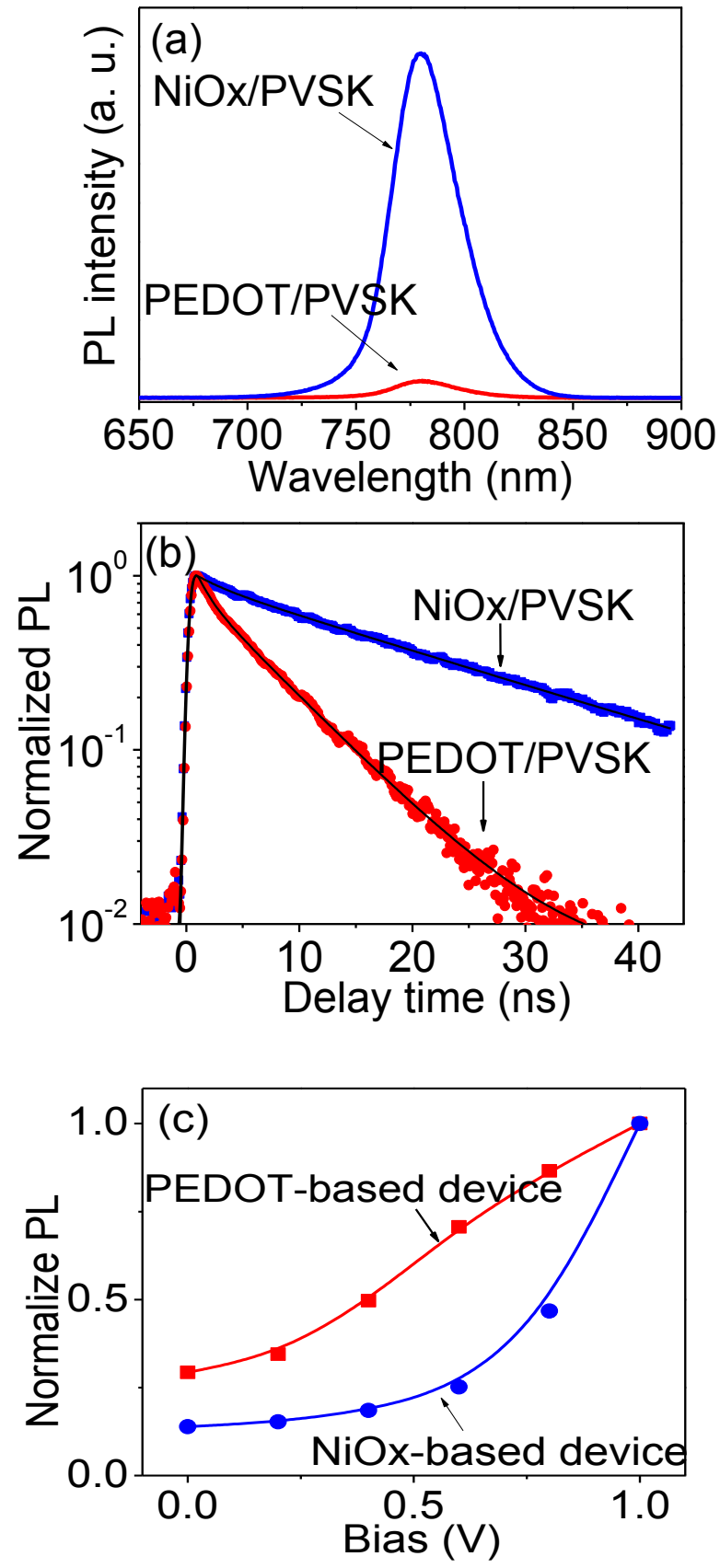

Fig. 5. a) PL spectra measured for NiOx/PVSK and PEDPT:PSS/PVSK double-layer samples. PVSK refers to the perovskite $\mathrm{CH}_{3} \mathrm{NH}_{3} \mathrm{PbI}_{3}$. b) Time-resolved PL lifetime for NiOx/PVSK and PEDPT:PSS/PVSK double-layer samples. The PL decay dynamics can be described by a fast component ( $\tau_{f}=1.26 n s$ and $A_{f}=0.34$ for PEDPT:PSS/PVSK sample, while $\tau_{f}=$ $2.18 n s$ and $A_{f}=0.14$ for NiOx/PVSK sample) and a slow component ( $\tau_{s}=6.59 n s$ and $A_{S}=0.88$ for PEDPT:PSS/PVSK sample, while $\tau_{s}=21.29 n s$ and $A_{s}=0.92$ for NiOx/PVSK sample). c) PL intensity is shown as a function of built-in potential by switching 
from short-circuit condition towards open-circuit condition in NiOx and PEDOT:PSS based devices.

Table 1. The photovoltaic parameters derived from current-voltage characteristics measured with forward and reverse scans for NiOx and PEDOT:PSS based devices.

\begin{tabular}{ccccc}
\hline Type of HTL & Jsc $\left(\mathrm{mA} / \mathrm{cm}^{2}\right)$ & Voc $(\mathrm{V})$ & FF & PCE $(\%)$ \\
\hline PEDOT:PSS(forward) & 19.32 & 0.88 & 0.75 & 12.80 \\
PEDOT:PSS(reverse) & 19.10 & 0.89 & 0.77 & 12.98 \\
NiOx(forward) & 21.14 & 1.01 & 0.75 & 16.01 \\
NiOx(reverse) & 21.21 & 1.00 & 0.75 & 16.91 \\
\hline
\end{tabular}




\section{Vitae}

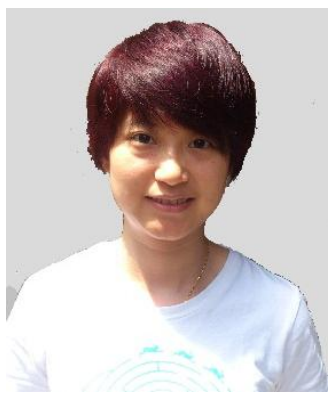

Pei-Ying Lin is currently pursuing her Ph.D. under the supervision of Prof. Tzung-Fang Guo in the Department of Photonics, National Cheng Kung University, Taiwan. She received her MS degree in the Department of Greenergy at National University of Tainan, Taiwan in 2012. Her current research focuses on device engineering and fundamental study in organo-metal halide perovskite-based hybrid solar cells

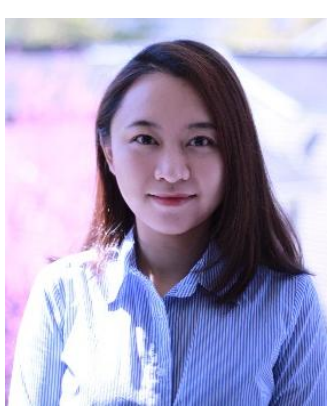

Ting $\mathrm{Wu}$ is currently pursuing her Ph.D. under the supervision of Prof. Bin $\mathrm{Hu}$ in the Department of Material Science and Engineering at the University of Tennessee, Knoxville, USA. She received her B.S. in polymer science (2009) and M.S. in materials science (2012) at Sichuan University, China. Her current research interests focus on materials development, device engineering and fundamental physics in perovskite solar cells.

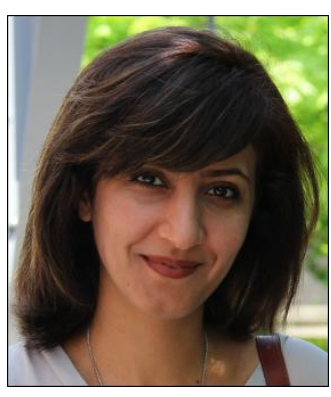

Dr. Mahshid Ahmadi is currently working as a research scientist at Joint Institute for Advanced Materials (JIAM), Department of Materials Science, the University of Tennessee, Knoxville, USA. She received her Ph.D. from Nanyang Technological University, Singapore in 2013, and then worked as a research technology consultant in a start-up company in Dallas, TX in 2014-2015. Her research interests have included materials development and electronic device fabrication. Specially, her current research focuses on organic-inorganic halide perovskite photovoltaics and high energy detectors. 


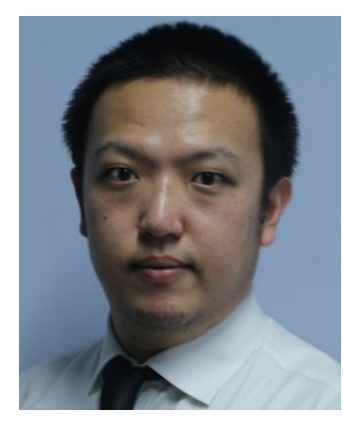

Li Liu is currently pursuing his Ph.D. in Physics under the supervision of Prof. Stefan Haacke in the University of Strasbourg, France. He received his M.S. in nanophotonics from the same university in 2013. His current research focuses on ultrafast spectroscopy study on the photophysics in different types of solar cells, including organic solar cells, dye-sensitized solar cells and perovskite solar cells.

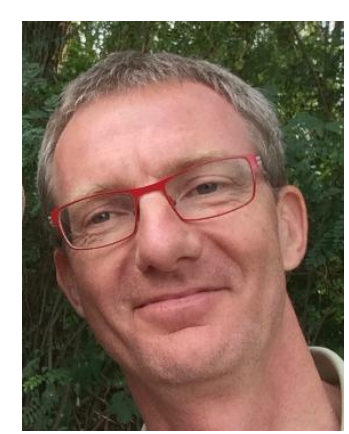

Stefan Haacke is a Professor of Physics at Strasbourg University and Director of the Strasbourg Institute of Physics and Chemistry of Materials (IPCMS). He obtained his $\mathrm{PhD}$ at J. Fourier University Grenoble, and worked 10 years at the Swiss Fed. Institute of Technology Lausanne (EPFL) in the groups of B. Deveaud and M. Chergui before joining Strasbourg University in 2004. His group develops femtosecond instrumentation for ultrafast spectroscopic studies of biomolecules, photo-receptor proteins, and organic or hybrid nanomaterials for energy applications.

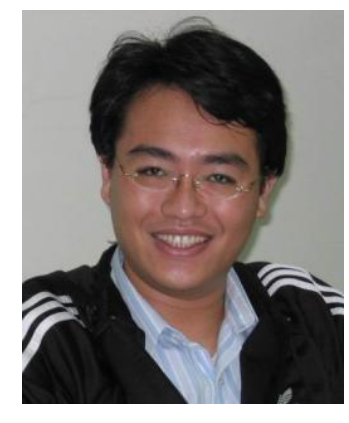

Professor Tzung-Fang Guo received Ph.D. in Materials Science and Engineering from University of California Los Angeles in 2002. Prof. Guo became the faculty at Department of Photonics, National Cheng Kung University, Taiwan in 2003 and served the Chairman since 2012. His research focuses on high-performance O/PLEDs, polymer PVs, ntype pentacene OTFTs, and the magnetic field effect of organic electronic devices. He also developed the perovskite-based hybrid solar cells of OPV $(p-i-n)$ configuration and applied ptype nickel oxide electrode interlayer in fabricating efficient perovskite-based hybrid solar cells and LEDs. 


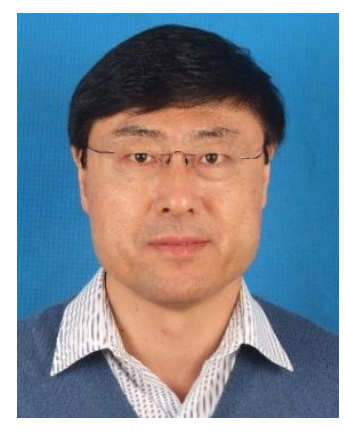

Professor Bin $\mathrm{Hu}$ received his Ph.D. from the Chinese Academy of Sciences under the supervision of Professor Xurong Xu in Condensed Matter Physics. He then worked as a postdoc at the Institute of Molecular Spectroscopy -- CNR, Bologna, Italy with Professor Carlo Taliani, and as research scientist at the University of Massachusetts/Amherst with Professor Frank Karasz. In 2002, he joined the faculty in the Department of Materials Science and Engineering at the University of Tennessee, Knoxville. His research includes spin-dependent processes in excited states, magneto-optic studies on photovoltaic and thermoelectric processes at different length scales, and lasing actions in ferroelectrically semiconducting materials. 


\section{Graphical Abstract}

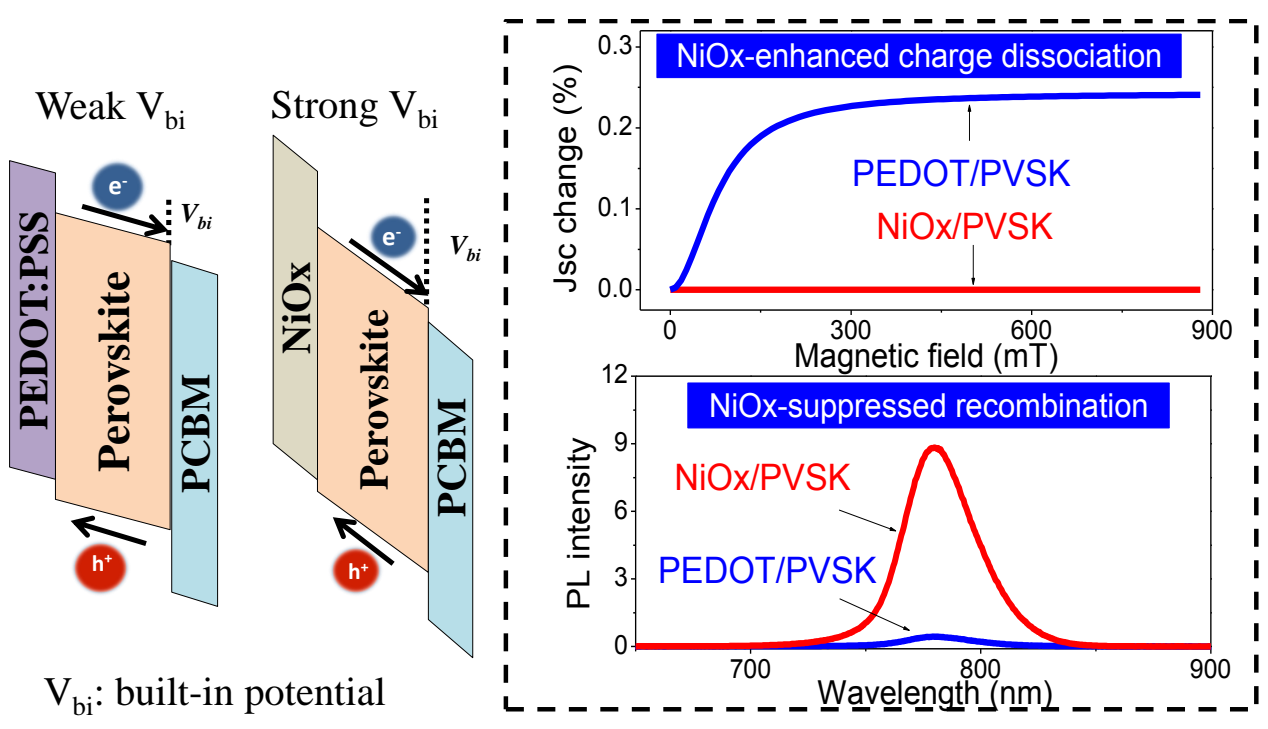

